\title{
Mutations in APOA5 or LDLR increase risk of myocardial infarction
}

In 1973, Goldstein and colleagues observed that survivors of early myocardial infarction (MI) had two common abnormalities in their lipid profiles: hypercholesterolaemia and hypertriglyceridaemia. Now, 40 years later in a paper published in Nature, investigators have identified rare mutations in two genes that support the observation that, in addition to the wellestablished contribution of a high LDLcholesterol level, disordered metabolism of triglyceride-rich lipoproteins is also a risk factor for MI.

Investigators in the Exome Sequencing Project coordinated by the National Heart, Lung, and Blood Institute in the USA identified 1,088 patients with MI at an early age (men aged $\leq 50$ years and women aged $\leq 60$ years) and a further 978 individuals of advanced age (men aged $\geq 60$ years and women aged $\geq 70$ years) who were free from MI, and sequenced the protein-coding regions of their genomes. After extensive statistical analysis and sequencing of several genes in an additional 6,721 patients with MI and 6,711 control individuals, the researchers found that carriers of a rare mutation in APOA5 (the gene encoding apolipoprotein A-V) had a 2.2-fold increased risk of MI compared with noncarriers. Carriers of rare nonsynonymous APOA5 alleles had a higher plasma triglyceride level $(167 \mathrm{mg} / \mathrm{dl}$ versus $105 \mathrm{mg} / \mathrm{dl} ; P=0.007)$ and a lower HDL-cholesterol level (43 mg/dl versus $57 \mathrm{mg} / \mathrm{dl} ; P=0.007$ ) than noncarriers. The LDL-cholesterol level did not significantly differ between the two groups. The investigators estimate that the burden of rare mutations in APOA5 explains approximately $0.14 \%$ of the total variance of MI and $0.28 \%$ of its heritability. The researchers believe that "the rare variant association signal presented [in their paper] establishes APOA5 as a bona fide MI gene".

In a second approach, the investigators sequenced the entire exome of additional patients and control individuals, bringing the total number of exomes analysed to 9,793 . With this sample size, rare alleles in only one gene, $L D L R$ (encoding the low-density lipoprotein receptor), conferred a risk of $\mathrm{MI}$ at exome-wide significance. Carriers of rare nonsynonymous mutations in $L D L R$ had a 4.2-fold increased risk of MI, whereas carriers of null alleles in $L D L R$ were at a 13-fold increased risk of MI, compared with noncarriers. Individuals with a disruptive mutation in $L D L R$ had a mean LDL-cholesterol level of $279 \mathrm{mg} / \mathrm{dl}$, whereas individuals with no nonsynonymous mutations in $L D L R$ had a mean LDL-cholesterol level of $135 \mathrm{mg} / \mathrm{dl}$. The investigators calculate that rare mutations in $L D L R$ account for $0.24 \%$ of the total variance of MI and $0.48 \%$ of its heritability. Approximately $2 \%$ of patients who experience an early MI have a rare, damaging mutation in $L D L R$.

In conclusion, the researchers highlight that "these data point to a route to MI beyond LDL cholesterol, namely triglyceride-rich proteins and the lipoprotein pathway. Genetic variation at two other proteins related to APOA5 function, apolipoprotein C-III [encoded by $A P O C 3$ ] and lipoprotein lipase [encoded by $L P L$ ], has been associated with triglycerides and MI risk." The investigators also observe that the discovery of rare variants that underlie complex diseases requires the genomic sequencing of thousands of individuals and subsequent, highly detailed statistical analysis. Such an extensive sample size is required because some diseaseconferring alleles are extremely rare, and these harmful alleles are not readily distinguishable from those associated with a benign prognosis.

Gregory B. Lim

Original article Do, R. et al. Exome sequencing identifies rare $L D L R$ and $A P O A 5$ alleles conferring risk for myocardial infarction. Nature doi:10.1038/nature13917

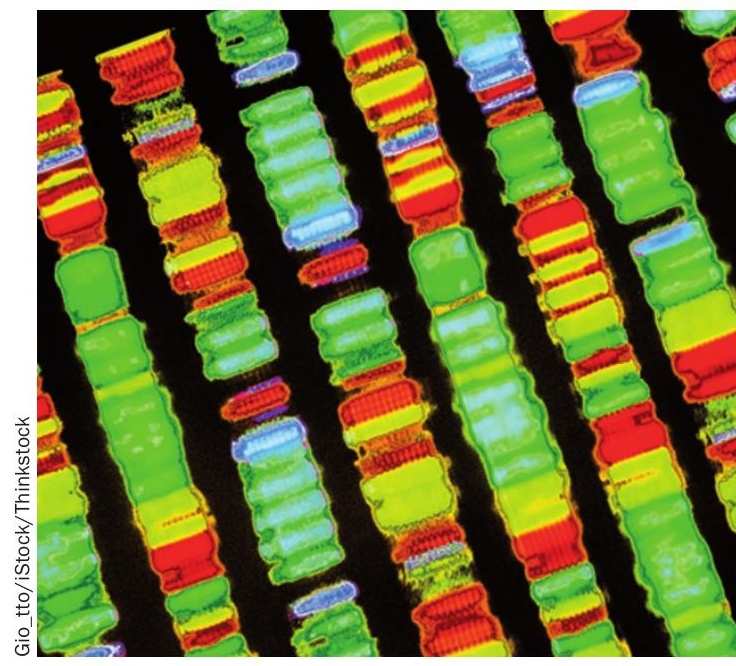

